\title{
Planetary nebulae as kinematic tracers of galaxy halos
}

\author{
Lodovico Coccato ${ }^{1}$ and PNS collaboration \\ ${ }^{1}$ European Southern Observatory, Karl-Schwarzschild-Str. 2, 85748 Garching b. München, \\ Germany \\ email:1coccato@eso.org
}

\begin{abstract}
The kinematics and dynamical properties of galaxy stellar halos are difficult to measure, given the faint surface brightness that characterizes these regions. Gas-rich systems such as spiral galaxies can be probed using the radio Hi emission. Early-type galaxies contain less gas, therefore alternative kinematic tracers need to be used. Planetary Nebulae (PNe) can be easily detected far out in the halo thanks to their bright [OIII] emission at $5007 \AA$. It is therefore possible to map the halo kinematics also in early-type galaxies, typically out to 5 effective radii or beyond. Thanks to the recent spectroscopic surveys targeting extra-galactic PNe, we can now rely on few tens of galaxies where the kinematics of the stellar halos are measured. I will discuss the most important results: (a) the relation of the stellar surface brightness and the PNe number density; (b) the velocity and velocity dispersion two-dimensional fields; (c) the radial profiles of angular momentum; and (d) the relation between the derived kinematics physical properties of the host galaxies.
\end{abstract}

Keywords. galaxies: general; galaxies: haloes; galaxies: kinematics and dynamics; ISM Planetary Nebulae: general

\section{Introduction}

Numerical simulations show that the galaxy formation mechanisms leave an imprint on the dynamic and kinematics of galaxies and their halos (e.g., Naab et al. 2014; Röttgers et al. 2014). Moreover, the dynamical time-scale of the halos (e.g., outside 2 effective radii, $R_{e}$ ) are of the order of few Gyr, therefore the imprints of the formation mechanisms are likely to be found in the orbital distribution of halo stars. In addition, the analysis of neutral gas revealed that the dark matter fraction increases with radius (e.g., Rubin et al. 1978). It is therefore extremely important to study the kinematics of galaxy halos to constrain galaxy formation and their dark matter content and distribution.

The faint stellar surface brightness level at large radii triggered the search for stellar kinematic tracers alternative to traditional absorption line spectroscopy. Planetary nebulae $(\mathrm{PNe})$, which are stars, and therefore somehow linked to the galaxy stellar component, can be detected at very high distances from the galaxy center thanks to their bright [OIII] emission line at $5007 \AA$. Other tracers such as OB stars or globular clusters are used with the same purpose, but they will not be discussed in this paper.

The exploitation of $\mathrm{PNe}$ as kinematic tracers and test particles for the gravitational potential started in the 80's (e.g., Nolthenius \& Ford 1987). In this framework, a dedicated instrument was build at the William Herschel Telescope in La Palma (Spain): the Planetary Nebulae Spectrograph (PN.S, Douglas et al. 2002).

In what follows, we summarize the main results from the PN.S survey (Section 2); also, we briefly discuss results from other surveys (Section 3). 


\section{The planetary nebulae early-type galaxy survey}

The PNS early-type galaxy survey aims at studying the dynamic and kinematic properties of early-type galaxies using PNe. It includes 33 galaxies ranging from E0 to E4 and S0, a $K$-band magnitude between -23 and -26 . Some spiral galaxies (e.g., M31, Merrett et al. 2006) has been also included to enlarge the original sample. In what follows we: i) describe the first analysis performed on the catalogs of positions, magnitudes, and velocities of the detected PNe (Section 2.1); and ii) present the most important observational results from this survey (Section 2.2).

\subsection{Analysis}

First, we clean the catalogs for: i) PNe that have low signal-to-noise ratio; ii) PNe that are most likely associated to a companion galaxy (where applicable), using a procedure that accounts for the PNe velocities and the surface brightness profiles of the galaxies (McNeil et al. 2010; Coccato et al. 2013); iii) kinematic outliers, using a friendless algorithm (Merrett et al. 2006) and a robust running $\sigma$ estimation (Longobardi et al. 2015a).

Second, we compute the completeness magnitude (i.e., the magnitude at which our sample is complete when considering only instrumental noise) and spatial completeness (how complete is our sample at a given distance from the galaxy center with respect to the completeness magnitude). Both quantities are computed using simulated images and sources that match the instrument set-up and the observed PNe luminosity function (see Coccato et al. 2009 for details). These two quantities are fundamental to compute the radial profile of PNe number density $\left(\Sigma_{\mathrm{PNe}}(R)\right)$, which will be directly compared to the stellar surface density profile. $\Sigma_{\mathrm{PNe}}(R)$ is computed by dividing the PNe sample into elliptical bins; in each bin we count the number of PNe brighter than the completeness magnitude and correct it for the spatial completeness in that elliptical bin.

Finally, we reconstruct the velocity and velocity dispersion two-dimensional fields from the individual PNe velocities along the line of sight. Indeed, the velocity of each $\mathrm{PNe}$ does not represent the mean of the line-of-sight velocity distribution (LOSVD) of the galaxy at that position. It is just a random velocity within the LOSVD in that position. Therefore, the galaxy LOSVD at a given position needs to be recovered by performing a statistical analysis of the PNe close to that position. This is done using an "adaptive Gaussian kernel smoothing" (Peng et al. 2004; Coccato et al. 2009).

\subsection{Results}

The reliability of the $\mathrm{PNe}$ as tracers for the underlying stellar population is confirmed by two observational evidences. First, the number density distribution of PNe follows reasonably well the surface brightness of the stellar component in the spatial region where these two components overlap (see Fig. 1). Second, the kinematics traced by the $\mathrm{PNe}$ is consistent with that of the stellar component in the regions were they overlap (see Fig. 2). These two results show that it is possible to combine the information of stars (that trace the inner regions) and $\mathrm{PNe}$ (that trace the outer regions) to get the entire kinematic and dynamical picture of a galaxy and its halo.

The initial results showed declining radial profiles of the velocity dispersion $(\sigma)$, which were interpreted as an indication of low dark matter content in these galaxies (e.g., Romanowsky et al. 2003; Méndez et al. 2009). Contrarily to these initial results, new dynamical models showed that these galaxies can arbor a dark matter halo consistent with $\Lambda \mathrm{CDM}$; their decreasing $\sigma$ profiles can be explained by strong radial anisotropy in the outer parts (de Lorenzi et al. 2009; Morganti et al. 2013). 

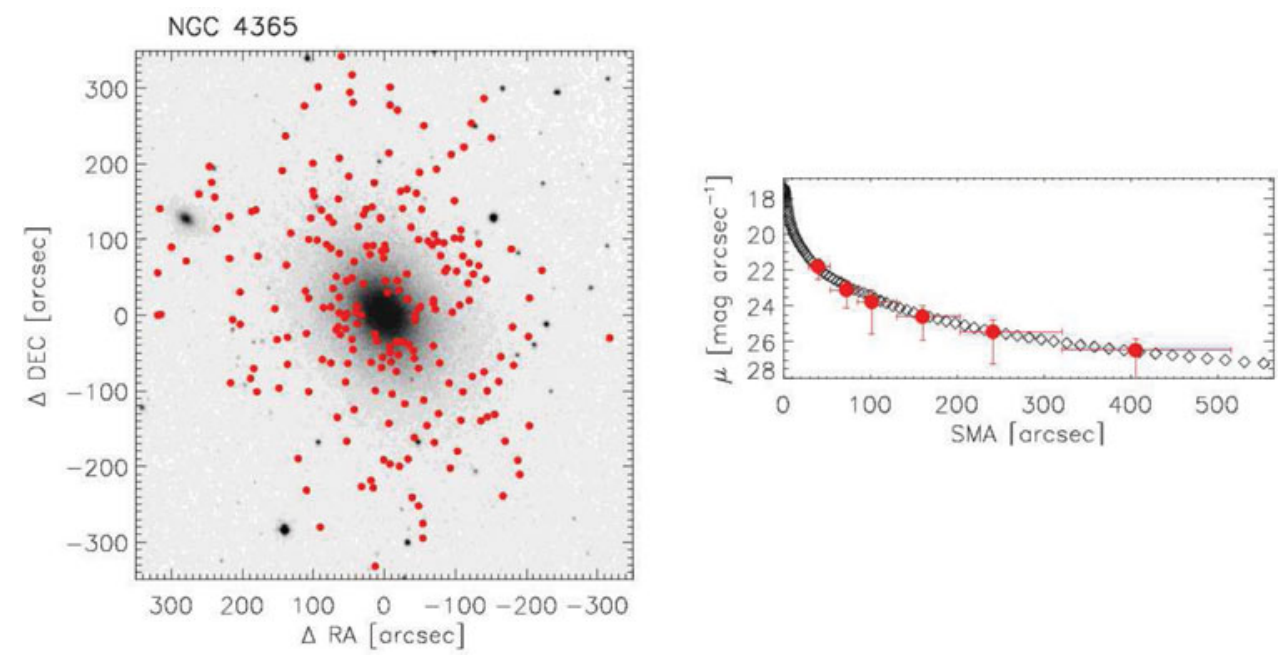

Figure 1. Spatial distribution of PNe (Arnaboldi et al., in preparation). Left panel: DSS image of NGC 4365; the detected PNe are marked in red. Right panel: comparison between the stellar surface brightness and $\Sigma_{\mathrm{PNe}}(R)=-2.5 \log _{10}\left[N_{\mathrm{PNe}}(R) / A(R) / c(R)\right]$, where $N_{\mathrm{PNe}}$ is the number of $\mathrm{PNe}$ within an elliptical annuls $R, A(R)$ is the area of the elliptical bin, and $c(R)$ is the completeness factor.

\subsubsection{Angular momentum}

The angular momentum of a galaxy can be represented by the $V / \sigma$ ratio or by the so-called $\lambda$ parameter (Emsellem et al. 2007). The former distinguishes between systems that are supported by "rotation" $(V / \sigma>>1)$ or by "pressure" $(V / \sigma<<1)$, whereas the latter is typically used to classify systems into "fast rotators" $(\lambda>0.1)$ and "slow rotators" $(\lambda<0.1)$. PNe trace the radial profiles of these two proxies out to 8-10 $R_{e}$. This represents a significant improvement with respect to previous studies based on absorption line spectroscopy, which were confined typically within 1 or $2 R_{e}$. In the innermost regions, the radial profiles of these proxies are either slowly rising (e.g., in slow rotators), or steeply rising and then flatten (e.g., in fast rotators), similar to the those of simulated galaxies ( Wu et al. 2014). In the halos however, their behavior can be different (e.g., Fig. 14 in Coccato et al. 2009). Some fast rotators have a profile that remains high and constant as in the inner regions (e.g., the stellar halo maintains the kinematic properties of the inner regions); in contrast, some fast rotators can show a rapid decline of $\lambda(R)$ (e.g., the stellar halo is more pressure supported that the inner regions). These differences between the central and outer regions can be the consequence of the different formation mechanism of the halos (e.g., Naab et al. 2014).

\subsubsection{Correlation with other galaxy properties.}

Another interesting result revealed by the PNe is that there exist correlations between the halo kinematics and other general physical properties of the galaxy. The halo kinematics is parametrized via two metrics, $\sigma_{\text {Last }}$, i.e., the outermost reached value of the velocity dispersion and Vrms $\mathrm{MIN}_{0.5}$, i.e., the minimum value of Vrms normalized at $0.5 R_{e}$; Vrms is defined as Vrms $=\sqrt{V^{2}+\sigma^{2}}$. Galaxies with small value of $\operatorname{Vrms}_{\mathrm{MIN} / 0.5}$ are those characterized by a decreasing $\operatorname{Vrms}(R)$ radial profile, galaxies with $\operatorname{Vrms}_{\mathrm{MIN} / 0.5} \sim 1$ are those characterized by a flat $\operatorname{Vrms}(R)$ radial profile.

Fig. 3 compares these two metrics with other galaxy properties. Galaxies with higher $\sigma_{\text {Last }}$ and flatter $\operatorname{Vrms}(R)$ profile are more luminous (in B-band and X-rays), have boxy 

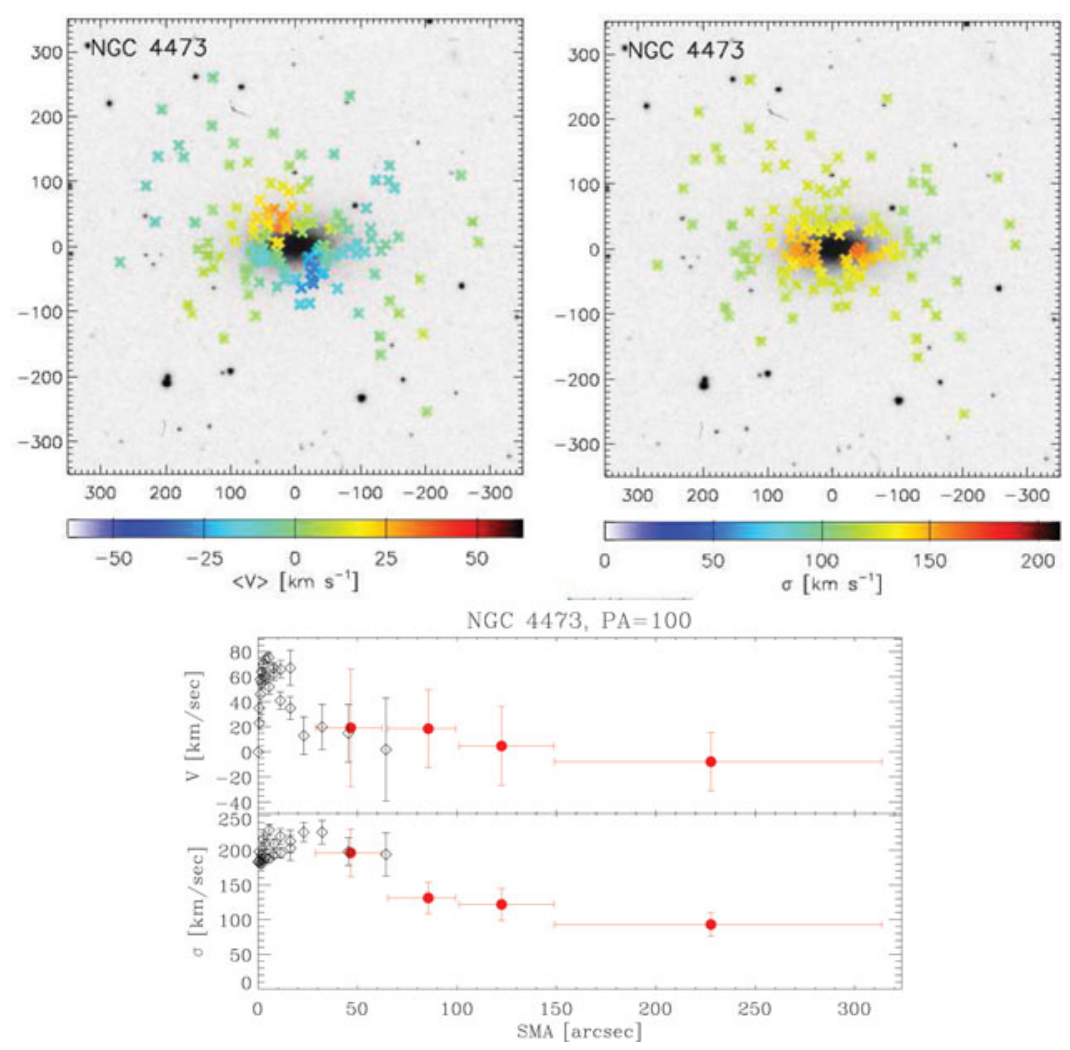

Figure 2. Kinematics of NGC 4473 as recovered using PNe (Pulsoni et al., in preparation). The top panels show the reconstructed two-dimensional fields of velocity (left) and velocity dispersion (right). Each cross represents the location of a PN, its color indicates the value of the reconstructed field in that position. The DSS image of NGC 4473 is shown in the background. The spatial scale is given in arcsec. North is up, East is left. The bottom panel compares the stellar kinematics (open diamonds, from Pinkney et al. 2003) along position angle $P A=100^{\circ}$ with that computed using the PNe within a cone along the same position angle (filled red circles).

isophotal profiles (a4), have smaller PNe specific frequency (the $\alpha$ parameter), and they are mostly pressure supported. Galaxies with lower $\sigma_{\text {Last }}$ and decreasing $\operatorname{Vrms}(R)$ radial profile are less luminous, have smaller PNe specific frequency, cover a wider range of isophotal shapes, and they are mostly rotationally supported.

\subsubsection{Tully-Fisher and Faber-Jackson scaling relations}

In S0 galaxies there are two main kinematic components: a disk supported by rotation and a spheroidal component supported by velocity dispersion. On a probabilistic basis, it is possible to divide the PNe into those that more likely belong to one or to the other component (Cortesi et al. 2011). This separation allowed Cortesi et al. (2013) to compare: i) the Tully-Fisher relation of stellar disks in S0s (determined using PNe associated to the disk) to that of spirals; and ii) the Faber-Jackson relation of stellar spheroids in S0s (determined using PNe associated to the spheroid) to that of ellipticals.

The results presented in Cortesi et al. (2013) show that the disks of S0s are dominated by rotation as those of spiral galaxies, although their velocity dispersion is higher than that of spirals. Also, S0s lie nearly one magnitude above the Tully-Fisher relation for spirals (S0s are one magnitude fainter than spirals with the same circular velocity). On the other hand, the spheroidal component of S0s is one magnitude brighter than 


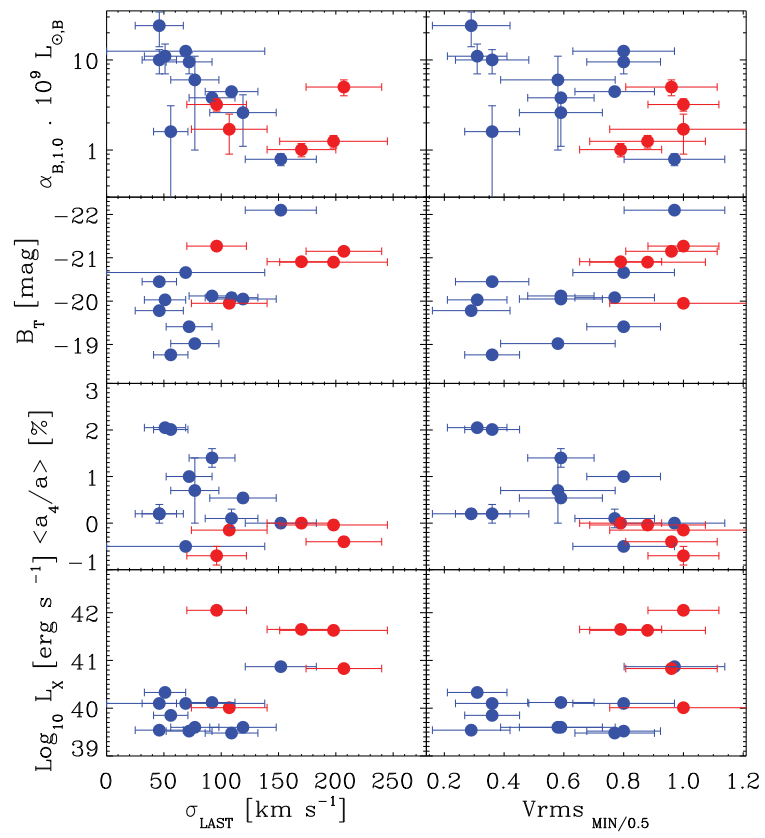

Figure 3. Correlation between halo kinematics and global galaxy properties. Left panels: value of the velocity dispersion at the outermost measured radius (generally outside $5 R_{e}$ ) versus other galaxy properties. From top to bottom: PNe specific frequency, total B-band luminosity, isophotal $a 4$ shape parameter, and total X-ray luminosity. Right panels: same as left panels but for the minimum value of Vrms normalized at $0.5 R_{e}$. Galaxies on the left side have declining Vrms profiles, galaxies on the right side have flat Vrms profiles. Blue and red symbols identify fast and slow rotators, respectively.

ellipticals with the same velocity dispersion. These findings suggest that spiral galaxies are "converted" into S0s through mild harassment: disks are heated, star formation is quenched, and spheroids are enhanced during the conversion process.

\section{Other PNe surveys}

Although the use of $\mathrm{PNe}$ as kinematic tracers was initially targeted to early-type galaxies to compensate for the lack of other tracers such as neutral gas like in spiral galaxies, several studies were also done on nearly face-on spirals. The aim was to measure the velocity dispersion orthogonal to the plane of the stellar disk $\left(\sigma_{z}\right)$. Herrmann \& Ciardullo (2009) studied a sample of 5 low-inclination spiral galaxies finding that:

- Stellar disks have a constant mass-to-light $(M / L)$ ratio out to 3 disk optical scale length, and that $\sigma_{z}$ decreases with radius. $\sigma_{z}$ flattens for outer radii. Explanations are either that i) $M / L$ increases at outer radii; or ii) the stellar disk is heated by halo substructures.

- Disks of early-type spirals have higher values of $M / L$ and are closer to maximal than the disks of late-type spirals.

- The inner halos of their sample galaxies are better fitted with a pseudo-isothermal law than a $\Lambda$ CDM profile.

PNe kinematics is also exploited to identify substructures in galaxy halos and intracluster light that probe interactions between galaxies and accretion events. It has been used for example, to probe the core of the Hydra I cluster (Ventimiglia et al. 2011), the 
halo of the Umbrella galaxy (Foster et al. 2014), M87 (Longobardi et al. 2015b), NGC 3311 (Ventimiglia et al. 2010), and those of spiral galaxies (e.g., Merrett et al. 2006; Herrmann et al. 2009; Fang et al. 2013).

\section{Conclusions}

Planetary nebulae are great kinematic tracers of the underlying stellar populations, as their distribution well trace the stellar light and their kinematics well agree with the stellar kinematics. They can be detected far out in the halo, extending considerably the boundaries reached by traditional absorption line spectroscopy. Thanks to the existing PNe surveys on elliptical, lenticular, and spiral galaxies we are now able to analyze the kinematic properties of stellar halos such as angular momentum, scaling relations, vertical velocity dispersion of stellar disks, and much more.

Acknowledgments. In memory of our collaborator and former P.I. of the PN.S project, Nigel Douglas. The Planetary Nebulae Team includes: M. Arnaboldi (ESO, Germany), M. Capaccioli (INAF-OAC, Italy), A. Chies-Santos (UFRGS, Brazil), L. Coccato (ESO, Germany), A. Cortesi (Sao Paulo University, Brazil), K. Freeman (RSAA, Australia), O. Gerhard (MPE-Garching, Germany), K. Kuijken (STRW-Leiden, The Netherlands), M. Merrifield (Nottingham, UK), R. Napolitano (INAF-OAC, Italy), C. Pulsoni (MPE, Germany), A. Romanowsky (UCO/Lick, USA), C. Tortora (INAF-OAC, Italy).

\section{References}

Coccato, L., Arnaboldi, M., \& Gerhard, O. 2013, MNRAS, 436, 1322

Coccato, L., Gerhard, O., Arnaboldi, M., et al. 2009, MNRAS, 394, 1249

Cortesi, A., Merrifield, M. R., Arnaboldi, M., et al. 2011, MNRAS, 414, 642

Cortesi, A., Merrifield, M. R., Coccato, L., et al. 2013, MNRAS, 432, 1010

de Lorenzi, F., Gerhard, O., Coccato, L., et al. 2009, MNRAS, 395, 76

Douglas, N. G., Arnaboldi, M., Freeman, K. C., et al. 2002, PASP, 114, 1234

Emsellem, E., Cappellari, M., Krajnović, D., et al. 2007, MNRAS, 379, 401

Fang, X., Zhang, Y., García-Benito, R., Liu, X.-W., \& Yuan, H.-B. 2013, ApJ, 774, 138

Foster, C., Lux, H., Romanowsky, A. J., et al. 2014, MNRAS, 442, 3544

Herrmann, K. A. \& Ciardullo, R. 2009, ApJ, 705, 1686

Herrmann, K. A., Ciardullo, R., \& Sigurdsson, S. 2009, ApJL, 693, L19

Longobardi, A., Arnaboldi, M., Gerhard, O., \& Hanuschik, R. 2015a, A\& A, 579, A135

Longobardi, A., Arnaboldi, M., Gerhard, O., \& Mihos, J. C. 2015b, A\&3A, 579, L3

McNeil, E. K., Arnaboldi, M., Freeman, K. C., et al. 2010, A\&AA, 518, A44

Méndez, R. H., Teodorescu, A. M., Kudritzki, R.-P., \& Burkert, A. 2009, ApJ, 691, 228

Merrett, H. R., Merrifield, M. R., Douglas, N. G., et al. 2006, MNRAS, 369, 120

Morganti, L., Gerhard, O., Coccato, L., Martinez-Valpuesta, I., \& Arnaboldi, M. 2013, MNRAS, 431,3570

Naab, T., Oser, L., Emsellem, E., et al. 2014, MNRAS, 444, 3357

Nolthenius, R. \& Ford, H. C. 1987, ApJ, 317, 62

Peng, E. W., Ford, H. C., \& Freeman, K. C. 2004, ApJ, 602, 685

Pinkney, J., Gebhardt, K., Bender, R., et al. 2003, ApJ, 596, 903

Romanowsky, A. J., Douglas, N. G., Arnaboldi, M., et al. 2003, Science, 301, 1696

Röttgers, B., Naab, T., \& Oser, L. 2014, MNRAS, 445, 1065

Rubin, V. C., Thonnard, N., \& Ford, Jr., W. K. 1978, ApJL, 225, L107

Ventimiglia, G., Arnaboldi, M., \& Gerhard, O. 2011, A\& A, 528, A24

Ventimiglia, G., Gerhard, O., Arnaboldi, M., \& Coccato, L. 2010, A\& A, 520, L9

Wu, X., Gerhard, O., Naab, T., et al. 2014, MNRAS, 438, 2701 